\title{
Rapid emergence of carbapenemase-producing Enterobacteriaceae isolates in Belgium
}

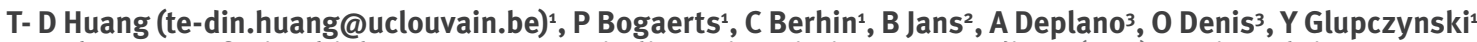

1. Laboratory of Microbiology, Centre Hospitalier Universitaire Mont-Godinne (UCL), Yvoir, Belgium

2. Epidemiology Unit, Scientific Institute of Public Health, Brussels, Belgium

3. Laboratory of Microbiology, Hôpital Erasme, Université Libre de Bruxelles, Brussels, Belgium

Citation style for this article:

Huang TD, Bogaerts P, Berhin C, Jans B, Deplano A, Denis O, Glupczynski Y. Rapid emergence of carbapenemase-producing Enterobacteriaceae isolates in Belgium. Euro Surveill. 2011;16(26):pii=19900. Available online: http://www.eurosurveillance.org/ViewArticle.aspx?Articleld=19900

Article published on 30 June 2011

We report on the evolution and epidemiology of carbapenem non-susceptible Enterobacteriaceae (CNSE) including carbapenemase-producing Enterobacteriaceae (CPE) in Belgium between January 2007 and April 2011. Significantly higher numbers of CNSE and of CPE were detected in 2010 and 2011 compared to the years 2007 to 2009. The majority of patients carrying CPE did not have history of travel abroad. The rapid emergence of autochthonous CPE strains in Belgium since 2010 warrants strengthened epidemiological surveillance at national level.

\section{Introduction}

Dissemination of Enterobacteriaceae that have acquired carbapenemase genes is a growing public health problem worldwide [1]. The prevalence of particular types of carbapenemases differs significantly between countries: Klebsiella pneumoniae carbapenemase (KPC) variants are prevalent in the United States, Greece, and Israel; Verona integron-encoded metallo-beta-lactamases (VIM) are frequently found in Greece and Italy; oxacillinase (OXA)-48 was first recovered and becoming prevalent in Turkey, while imipenemase (IMP)-type enzymes were mostly reported in the Far East [2]. Recently, isolates with New Delhi metallo-beta-lactamases (NDM-1) have been identified, mostly in patients who had a history of travel to the Indian subcontinent [3].

In Belgium, sporadic cases of carbapenemase-producing Enterobacteriaceae (CPE) isolates have been reported, the majority of which occurred in patients returning from travel abroad [4-6].

This longitudinal survey aimed to summarise the microbiological characteristics and clinical data of carbapenem-non-susceptible Enterobacteriaceae (CNSE) isolates, with a special focus on CPE isolates referred to the National Reference Centre (NRC) for multidrugresistant Enterobacteriaceae.

\section{Methods}

Enterobacteriaceae isolates were referred by local Belgian laboratories on a voluntary basis to the NRC
(Centre Hospitalier Universitaire Mont-Godinne, Yvoir, Belgium). They were invited to send isolates that, according to antimicrobial susceptibility guidelines used in the respective laboratories, were resistant to third or fourth generation cephalosporins or carbapenem nonsusceptible, for detection or confirmation of enzymes conferring antibiotic resistance. In September 2008, following the detection of the first VIM-1-producing Klebsiella pneumoniae isolates causing nosocomial outbreaks in two Belgian hospitals [7], a national alert was issued and local laboratories were asked to send carbapenem-non-susceptible isolates to the NRC for the detection or confirmation of carbapenemase production. All isolates referred between Janurary 2007 and April 2011 were re-tested at the NRC for antimicrobial susceptibility by the disk diffusion method according to the guidelines of the Clinical and Laboratory Standards Institute (CLSI) and meropenem inhibition zones were recorded. Isolates displaying an inhibition zone of <23 $\mathrm{mm}$ to $10 \mu \mathrm{g}$ meropenem disks, categorised retrospectively as intermediately resistant according to the CLSI interpretative criteria of January 2011 [8], were screened for the presence of carbapenemase-encoding genes by PCR using primers specific for bla VIM, bla IMP, bla KPC, blaNDM and blaOXA-48 [6]. Carbapenemase-encoding genes were identified by sequencing of the amplicons. Clinical and demographic data, including recent travel and/or hospitalisation abroad, were collected for all patients carrying CPE strains.

\section{Results}

A total of 155, 133, 125, 93 and 49 Enterobacteriaceae isolates were referred to the NRC during the years 2007, 2008, 2009, 2010 and the first four months of 2011, respectively. Numbers and the respective proportions of CNSE (including CPE) isolates referred during the study period are shown in Figure 1. The proportion of CNSE increased significantly from 8\% (33 of 413 isolates) in the years 2007 to 2009 to $50 \%$ (71 of 142 isolates) in the years 2010 and 2011 (p<0.0001). The total number of CPE isolates we detected was also significantly higher in $2010(n=17)$ and $2011(n=13)$ than in the years 2007 to 2009 ( $p<0.0001)$. 
The evolution over time and the microbiological characteristics of the $44 \mathrm{CPE}$ isolates detected during the study period are shown in Figures 2, 3 and 4. VIM-1 $(n=18)$ and OXA-48 $(n=16)$ were the most frequent carbapenemase enzymes found among CPE isolates.

Of the 44 different CPE isolates recovered from 39 different patients at 16 Belgian hospitals during the study period, 20 were isolated from urine, nine from rectal screening swabs (for the detection of asymptomatic carriers), seven from wound/drainage fluids, six from lower respiratory tract samples and three from blood culture specimens.

\section{FIGURE 1}

Evolution of number and proportion of carbapenemnon-susceptible Enterobacteriaceae isolates including carbapenemase producers, National Reference Centre, Belgium, January 2007-April $2011(\mathrm{n}=555)$

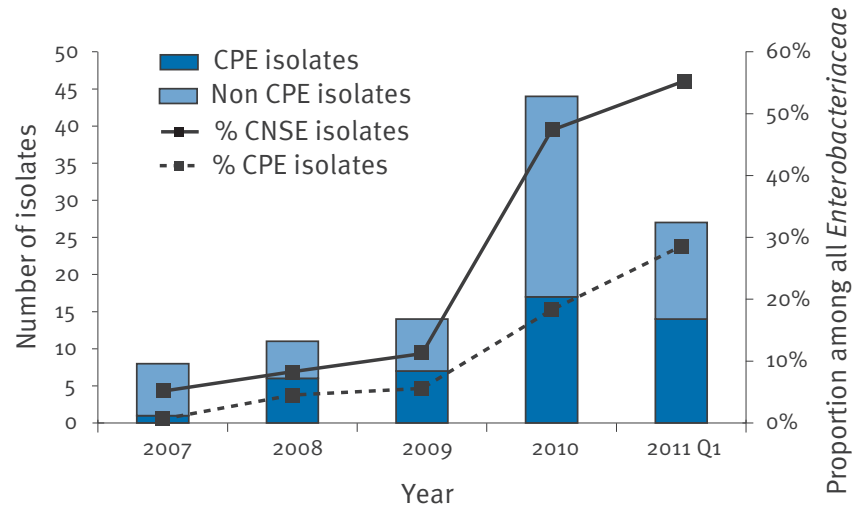

CNSE: carbapenem-non-susceptible Enterobacteriaceae; CPE: carbapenemase-producing Enterobacteriaceae; Q1: first quarter.
Of the 39 patients colonised or infected with a CPE isolate, only 16 had travelled abroad (13 of them in Mediterranean countries of Europe or North Africa) in the six months before the diagnosis (Table). Eleven of the 23 autochthonous CPE cases, probably resulting from local secondary acquisition, carried VIM-1positive isolates (seven Klebsiella pneumoniae, two Serratia marcescens and two K. oxytoca) and were identified in eight hospitals. The 12 remaining patients harboured OXA-48-producing isolates (seven Enterobacter cloacae, four K. pneumonia and 1 Escherichia coli) and were detected in four hospitals. Four patients harboured more than one CPE isolates. Two patients were colonised by two NDM-1-positive isolates of two different species: one of them carried $K$. pneumoniae associated with E. coli and the other patient Morganella morganii with $E$. cloacae. One patient was colonised by two OXA-48-producing isolates (one E. cloacae

\section{FIGURE 3}

Species and resistance genes distribution of carbapenemaseproducing Enterobacteriaceae isolates, National Reference Centre, Belgium, January 2007-April 2011 ( $\mathrm{n}=44$ )

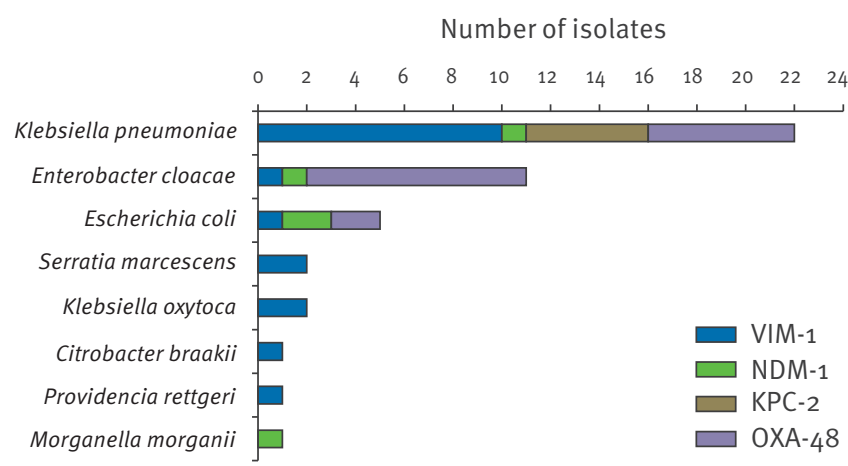

KPC: Klebsiella pneumoniae carbapenemase; NDM: New Delhi metallo-beta-lactamase; OXA: oxacillinase; VIM: Verona integronencoded metallo-beta-lactamase.

\section{FIGURE 4}

Evolution of the species distribution of carbapenemaseproducing Enterobacteriaceae isolates, National Reference Centre, Belgium, January 2007-April $2011(n=44)$

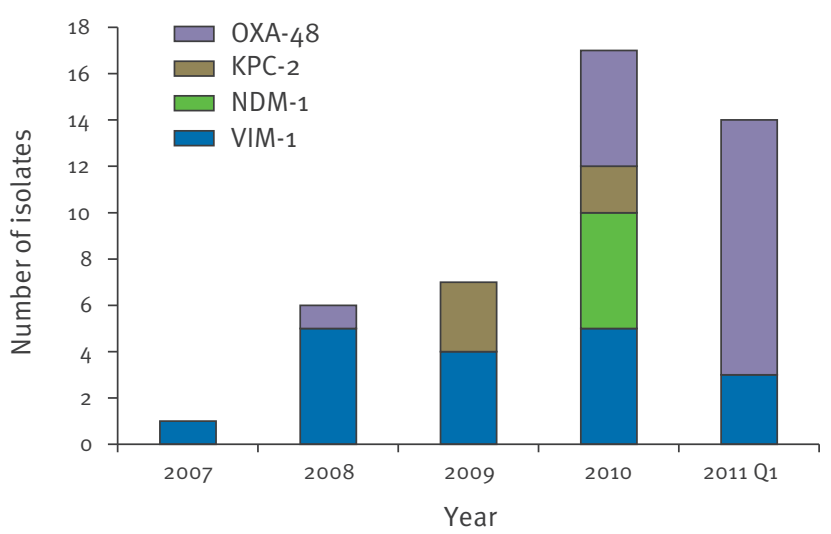

KPC: Klebsiella pneumoniae carbapenemase; NDM: New Delhi metallo-beta-lactamase; OXA: oxacillinase; Q1: first quarter; VIM: Verona integron-encoded metallo-beta-lactamase.

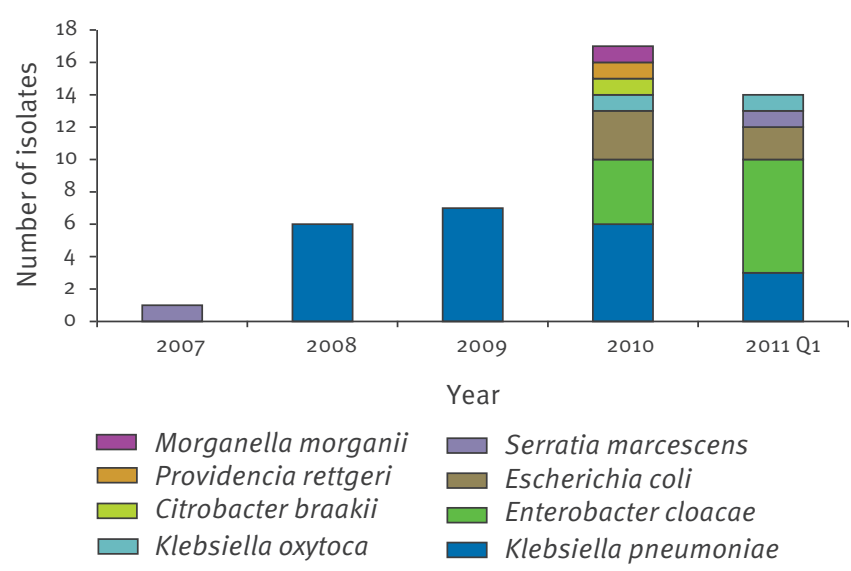

Q1: first quarter. 
and one E. coli). In one patient one VIM-1-producing Providencia rettgeri, one VIM-1-positive Citrobacter freundii and one KPC-2-producing $K$. pneumoniae were detected simultaneously. Retrospective review of clinical data charts showed that 25 patients were considered colonised by CPE isolates while 14 patients were considered to be infected. The infections were urinary tract infections $(n=7)$, lower respiratory tract infections $(n=2)$, soft-tissue and skin infections $(n=2)$ and blood stream infections $(n=3)$.

\section{Discussion}

Our data clearly demonstrate a significant increase of CNSE isolates referred to the NRC and of CPE isolates detected in Belgian hospitals particularly since 2010. While for the period from 2007 to 2009 almost all carbapenemase-producing isolates were $K$. pneumoniae, eight different Enterobacteriaceae species were detected from 2010 onwards. Of note, OXA-48 CPE isolates emerged since 2010 and have now become the predominant carbapenemase in CPE isolates causing nosocomial outbreaks at two different hospitals in Belgium during the first quarter of 2011 (unpublished data). Vaux et al. reported similar trends of emergence of CPE isolates in France since 2010, and OXA-48 carbapenemase represented the majority of the resistance mechanism in CPE isolates in France as well [9].

In Belgium, no resistance survey of Enterobacteriaceae isolates with decreased susceptibility to carbapenems has been conducted to date and thus no data are available regarding the true prevalence of CNSE isolates. It is possible that a certain number of CNSE and CPE isolates were missed, especially those producing OXA-48 and lacking expanded-spectrum cephalosporin-hydrolysing beta-lactamase [10].

It is presently unclear whether the apparent increase in the isolation of CNSE and CPE is due to the spreading potential of these microorganisms or rather reflects the application of more effective detection methods by clinical laboratories. The decreasing number of total isolates referred to NRC from 2007 to 2010 cannot be explained, but together with the significantly increasing number of CNSE isolates referred, could result in the relative increase of CNSE or CPE. Since that the isolates were referred on a voluntary basis, a better knowledge or application of detection methods for ESBL-carrying or AmpC cephalosporinase-producing strains by local laboratories could result in a decreased need for confirmation and referral of these isolates to the NRC. It is possible as well that the adherence to the EUCAST breakpoints or to the revised CLSI interpretative criteria for carbapenems published in June 2010 [11] could have improved the detection of CNSE and of CPE isolates by clinical laboratories. The increased sensitivity of detection, together with a better awareness of clinical microbiologists, might in part explain the higher number of CNSE isolates referred to the NRC since 2010. Nevertheless, the diversification of both the species and the carbapenemase-encoding genes detected in CPE isolates is a matter of concern. Moreover, the significant proportion of non-travel associated CPEpositive patients suggests the local establishment and spread of carbapenemase-producing enterobacteria in Belgium. These data clearly underline the importance of a systematic and coordinated national surveillance programme for rapid detection and reporting of CPE isolates in order to prevent their further dissemination. Finally, our experiences suggest that at a European level, the implementation of harmonised and accurate detection methods should be encouraged and that specific guidelines of management including infection control measures are urgently needed in order to limit the spread of these multidrug-resistant organisms.

\section{References}

1. Cornaglia G, Rossolini GM. The emerging threat of acquired carbapenemases in Gram-negative bacteria. Clin Microbiol Infect. 2010;16(2):99-101.

2. Queenan AM, Bush K. Carbapenemases: the versatile -lactamases. Clin Microbiol Rev. 2007;20(3):440-58.

3. Kumarasamy KK, Toleman MA, Walsh TR, Bagaria J, Butt F, Balakrishnan $\mathrm{R}$, et al. Emergence of a new antibiotic resistance mechanism in India, Pakistan, and the UK: a molecular, biological, and epidemiological study. Lancet Infect Dis. 2010;10(9):597-602.

4. Cuzon G, Naas T, Bogaerts P, Glupczynski Y, Huang TD, Nordmann P. Plasmid-encoded carbapenem-hydrolyzing beta-lactamase OXA-48 in an imipenem-susceptible Klebsiella pneumoniae strain from Belgium. Antimicrob Agents Chemother. 2008;52(9):3463-4.

5. Bogaerts P, Montesinos I, Rodriguez-Villalobos H, Blairon L, Deplano A, Glupczynski Y. Emergence of clonally related Klebsiella pneumoniae isolates of sequence type 258 producing KPC-2 carbapenemase in Belgium. J Antimicrob Chemother. 2010;65(2):361-2.

6. Bogaerts P, Bouchahrouf W, de Castro RR, Deplano A, Berhin C, Piérard D, et al. Emergence of NDM-1-producing Enterobacteriaceae 1 in Belgium. Antimicrob Agents Chemother. 2011;55(6):3036-8.

7. Glupczynski Y, Rodriguez-Villalobos H, Bogaerts P, Blairon L, Gérard M, Aoun M, et al. Emergence of pan-resistant VIM1 producing Klebsiella pneumoniae in Belgian hospitals. Abstract presented at the 19th European Congress of Clinical Microbiology and Infectious Diseases, Helsinki, Finland. 2009. Abstract no $\mathrm{P}_{1692 .}$

8. Clinical and Laboratory Standards Institute (CLSI). Performance Standards for Antimicrobial Susceptibility Testing. Twentieth Informational Supplement M100-S21. Wayne, Pennsylvania: CLSI; 2011.

9. Vaux S, Carbonne A, Thiolet JM, Jarlier V, Coignard B, RAISIN and Expert Laboratories Groups. Emergence of carbapenemase-producing Enterobacteriaceae in France, 2004 to 2011. Euro Surveill. 2011;16(22):pii=19880. Available from: http://www.eurosurveillance.org/ViewArticle. aspx?Articleld $=19880$

10. Carrer A, Fortineau N, Nordmann P. Use of ChromID extended-spectrum beta-lactamase medium for detecting carbapenemase-producing Enterobacteriaceae. J Clin Microbiol. 2010;48(5):1913-4.

11. Clinical and Laboratory Standards Institute (CLSI). Performance Standards for Antimicrobial Susceptibility Testing. Twentieth Informational Supplement M100-S20-June Update. Wayne, Pennsylvania: CLSI; 2010. Available from: http://www.clsi.org/ source/orders/free/m100-s20-u.pdf 